\title{
Portal vein embolization and application of autologous stem cells in patients with primary unresectable liver tumours
}

\author{
Treska V ${ }^{1}$, Koza V², Lysak D², Slauf $F^{3}$, Mirka $H^{3}$, Sutnar A ${ }^{1}$, Skalicky $T^{1}$, Fichtl $J^{1}$, Ferda $J^{3}$ \\ Clinic of Surgery of the Faculty Hospital and Medical Faculty of Charles University in Pilsen, Czech Republic. \\ treska@fnplzen.cz
}

\begin{abstract}
Background: Only 15-20\% of patients with liver tumours can undergo radical surgery. Insufficient future liver remnant volume (FLRV) is one of the main causes of tumours unresectability. Portal vein embolization (PVE) together with administration of haematopoietic stem cells (HSC) may expand the operability of primary unresectable liver tumours.

Methods: In this pilot study, the authors reported on five patients (1 hepatocellular carcinoma, 4 colorectal cancer metastases) with FLRV $<30 \%$, who underwent PVE on the side of the tumour with a subsequent application of HSC to the non-embolized branch of portal vein.

Results: PVE with HSC application was without any complications. In three patients, a sufficient increase of FLRV occurred within 2-4 weeks followed by a liver resection. All patients were between 5-12 months after the surgery in good condition; one of them was diagnosed with pulmonary metastasis after nine months that was successfully treated with laser metastasectomy. In one patient with hepatocellular carcinoma, an increase of FLRV and progression of the tumour in the liver occurred following the PVE with administration of HSC and the patient was treated only symptomatically. Despite an adequate increase of FLRV, severe intraabdominal adhesions hampered liver resection in one patient.

Conclusions: Combination of PVE with HSC administration appeared to be a promising method that stimulated growth of FLRV with a subsequent possibility of an early radical liver resection. The issue is a danger of tumour progression in the liver parenchyma following the PVE with HSC. The current randomized study should answer these questions (Tab. 1, Fig. 4, Ref. 38). Full Text in PDF www.elis.sk.

Key words: unresectable liver tumours, portal vein embolization, haematopoietic stem cells.
\end{abstract}

To date, liver resection has been the only radical therapy in patients with primary or secondary liver tumours. Unfortunately, the liver tumour is radically resectable only in $15-20 \%$ of patients at the time of diagnosis. An insufficient liver tissue volume following the resection, so called future liver remnant volume (FLRV), is one of the main causes of inoperability of liver tumours. In these cases, portal vein embolization (PVE) on the side of the tumour is an important step to increase the volume of the contralateral liver lobe with a subsequent liver resection. An increase of sufficient liver volume needed for resection occurs usually within 4 to 6 weeks after the PVE. However, in some patients, from various commonly unclear reasons, no increase of the required FLRV occurs so that they could undergo a liver resection. Tumour growth or its dissemination in the body is another problem in some patients following the PVE.

${ }^{1}$ Clinic of Surgery of the Faculty Hospital and Medical Faculty of Charles University in Pilsen, ${ }^{2} \mathrm{Hematooncological}$ Department of the Faculty Hospital in Pilsen, and ${ }^{3}$ Radiodiagnostic Clinic of the Faculty Hospital and Medical Faculty of Charles University in Pilsen, Czech Republic

Address for correspondence: V. Treska, MD, PhD, Department of Surgery, University Hospital, Alej Svobody 80, CZ-304 60 Pilsen, Czech Republic.

Phone: +420.37 .7104270$

Acknowledgements: The study was supported by the grants of Charles University, Prague, P36.
Therefore, the aim of this study was to find out the means, which would accelerate growth of the healthy liver lobe following the PVE and reduce the time period needed for hypertrophy of the liver tissue following the PVE as much as possible. Potential growth promoters of liver tissue after the PVE may include hematopoietic stem cells (HSC), which normally participate in the regeneration of liver tissue (1). The authors present their first clinical experience with a selective application of autologous HSC CD 34+ and CD 133+ combined with PVE in patients with primary inoperable liver tumours.

\section{Methods}

In this prospective non-randomized trial (initiated on May 1, 2009) we administered autologous HSC CD34+ and CD 133+ to five patients (4 patients with metastatic colorectal cancer, one with hepatocellular carcinoma) The study included patients with a primary inoperable liver tumour because of insufficient FLRV, in whom no extrahepatic tumour metastases were found (ultrasoundUSG, computed tomography - CT, positron emission tomography - PET-CT). As an insufficient FLRV, we considered the volume below $30 \%$ of the total volume of the liver tissue, because all patients with liver metastases indicated for liver resection underwent within a short period of time before the intended liver resection chemotherapy or chemotherapy combined with biological therapy due to the primary tumour. 
Tab. 1. Characteristic of the group of patients.

\begin{tabular}{|c|c|c|c|c|c|}
\hline Patient No & 1 & 2 & 3 & 4 & 5 \\
\hline Age/sex & $54 / \mathrm{F}$ & $75 / \mathrm{M}$ & $55 / \mathrm{F}$ & $60 / \mathrm{M}$ & $65 / \mathrm{M}$ \\
\hline Diagnosis & $\mathrm{HCC}$ & CLM & CLM & CLM & CLM \\
\hline Brunt & G0S0 & G0S0 & G0S0 & G1S1 & G0S0 \\
\hline Ishak & G0S0 & G1S0 & G1S0 & G0S1 & $\mathrm{G} 2 \mathrm{~S} 0$ \\
\hline TLV before PVE (ml) & 2610 & 1777 & 866 & 1953 & 1500 \\
\hline FLRV before PVE (ml/\%) & $384 / 14.7$ & $450 / 25.3$ & $218 / 25.1$ & $585 / 29.9$ & $403 / 26.9$ \\
\hline FLRV before surgery (ml/\%) & $734 / 28.1$ & $829 / 46.6$ & $421 / 48.6$ & $710 / 36.3$ & $634 / 42.3$ \\
\hline Apheresis product volume $(\mathrm{ml})$ & 103 & 114 & 118 & 114 & 131 \\
\hline Applied CD133+ (x 106) & 112.9 & 52.3 & 20.6 & 122.2 & 12.0 \\
\hline Applied CD34+ $\left(\times 10^{6}\right)$ & 146.4 & 66.3 & 29.6 & 166.6 & 31.8 \\
\hline Liver hypertrophy (weeks) & 4 & 3 & 4 & 3 & 3 \\
\hline Tumour volume before PVE (ml) & 792 & 257 & 86 & 532 & 98 \\
\hline Tumour volume after PVE (ml) & 967 & 234 & 83 & 675 & 110 \\
\hline Liver surgery & No (tu progression) & Yes & Yes & No (adhesions) & Yes \\
\hline
\end{tabular}

TLV - total liver volume, FLRV - future liver remnant volume, ICG - indocyanine green test, PVE - portal vein embolization

One female and four males of the mean age of 61.2 years $(54$ 75 years) were concerned. We performed an initial volumometry of the liver and determined the FLRV (Somatom Definition, Siemens, Germany). The mean initial FLRV was $24.4 \%$. The function of the liver parenchyma was evaluated by clinical and laboratory parameters and using the retention test of indocyanine green (Limon, Pulsion Medical Systems AG, Munich, Germany). Patients under ultrasound control also underwent a liver parenchyma biopsy outside the tumour that was assessed by a histopathologist according to Brunt and Ishak criteria $(1,2)$ to clarify a histopathological degree of liver parenchyma impairment. In the indication criteria, we followed the generally applicable rules for the FLRV prior to liver resection with regard to function and morphology of the liver tissue $(2,3)$. Neoadjuvant chemotherapy targeted at the primary or secondary liver tumour was not performed. All patients were informed about the proposed treatment process in detail and were advised of potential risks (especially further tumour progression) and they signed an informed consent form.

The source of stem cells were peripheral hematopoietic stem cells (HSC) collected by means of apheresis from peripheral blood. The patients were stimulated with granulopoesis growth factor (GCSF, Neupogen, Amgen Europe B.V., Breda, The Netherlands) at a dose of $10 \mu \mathrm{g} / \mathrm{kg} /$ day administered subcutaneously in one daily dose for four days. From the fourth day following the administration of Neupogen, the circulating stem cells were monitored in peripheral blood (detected by the flow cytometry as CD34+ cells). On the fifth day after the administration of Neupogen, one large-volume leukopheresis was performed via the dialysis catheter inserted into the femoral vein using the continuous high-volume apheresis blood element separator Cobe Spectra (CaridianBCT, Lakewood, CO, USA), the program for collection of mononuclear leukocytes (MNC program, software version 6.1). Approximately 3 volumes of each patient's blood were processed, and citrate- and citric acid-based solution was used as an anticoagulant (ACD-A, Baxter, Deerfield, IL, USA) in the ratio 1:12 to 1:14 in the whole blood. To prevent citrate toxicity, all patients underwent calcium supplementation into the efferent line (in fractions, total dose 10 to $20 \mathrm{~mL} \mathrm{CaCl}_{2}$ ). The yield product was analyzed in the laboratory and the basic quality parameters were established: volume, concentration and absolute leukocyte and CD34+ count, erythrocyte and thrombocyte count, variability of CD34+ cells, sterility, CD133+ cell content (Tab. 1). Collection of cells for determination of all tests was performed in the closed system. Prior to mobilization, all patients underwent an examination to exclude blood-transmitted diseases. No further manipulation was performed with the product and it was stored till the next day at $2-8{ }^{\circ} \mathrm{C}$ under a continual monitoring of storage conditions. The product was provided from the laboratory for implantation in the operating room the next day.

One day before the leukapheresis, we performed the PVE transparietally using the Histoacryl mixture (BBraun, Germany): Lipiodol (Cedex, France) diluted in the 1:10 ratio (Fig. 1). On the following day after leukapheresis, we performed a small laparotomy in the right hypogastrium and inserted a catheter through the ileocolic vein to the contralateral branch of the portal vein under a general anaesthesia and administered $100 \mathrm{~mL}$ of the acquired prod-

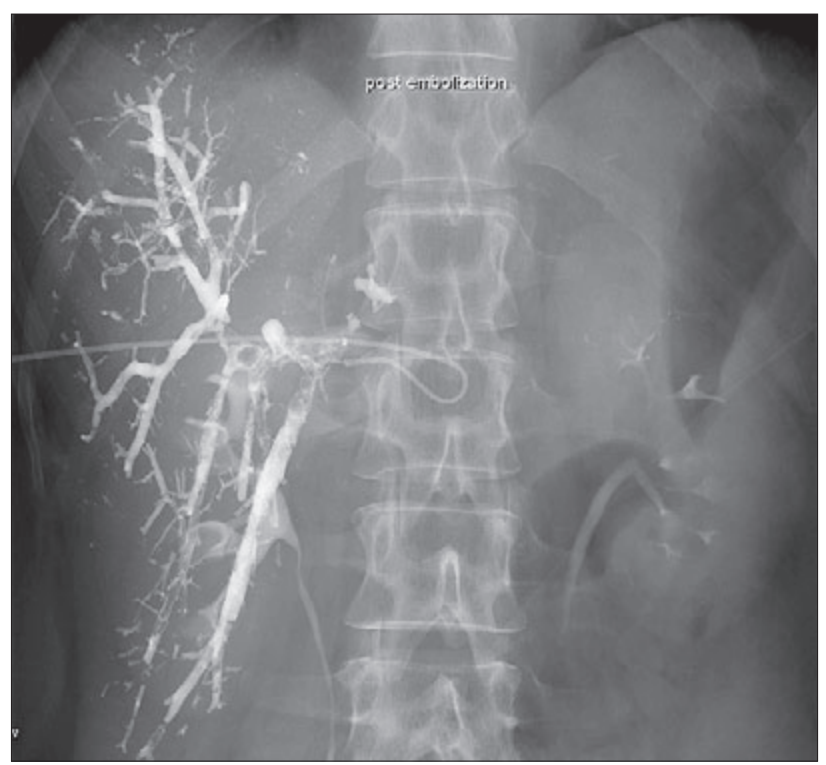

Fig. 1. PVE of the right liver lobe. 


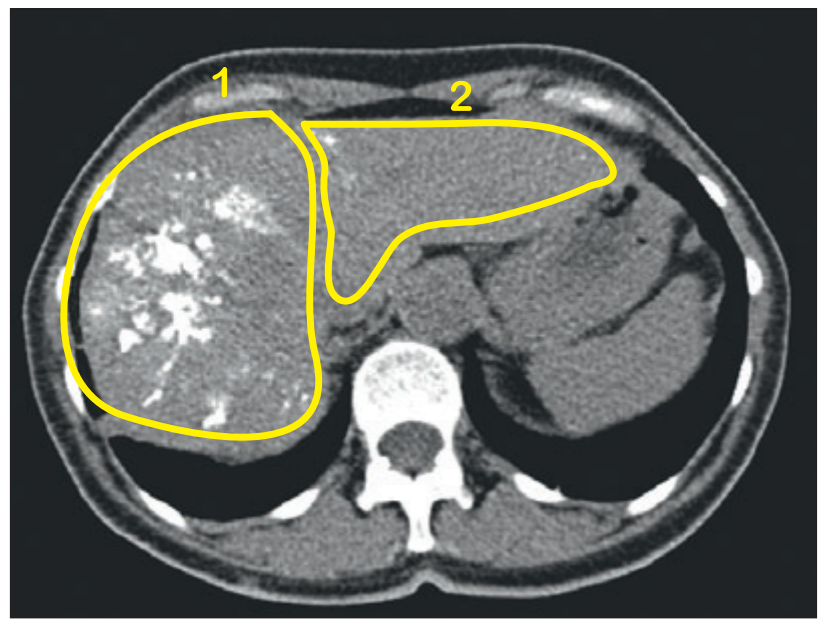

Fig. 2. Sufficient FRLV two weeks after PVE and application of HSC.

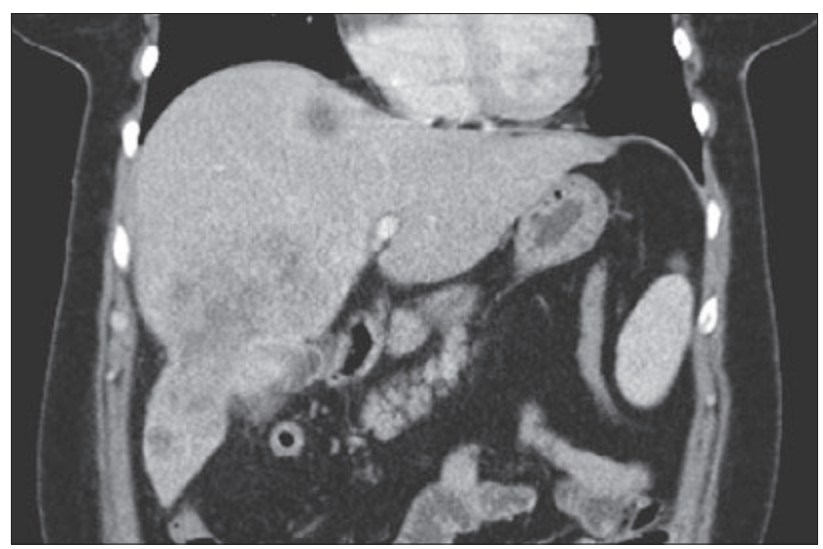

Fig. 3. HCC in the right liver lobe prior to PVE and application of HSC.

uct with a minimum required volume of $1 \times 10^{7} \mathrm{CD} 133+\mathrm{HSC}$. We monitored the growth of the contralateral liver lobe in both groups using the CT volumometry of the liver in weekly intervals following the PVE or administration of HSC. As soon as a sufficient growth of the volume of the contralateral liver lobe was achieved (Fig. 2), we performed a liver resection. If the tumour progressed in the liver (measured again using the CT volumometry) or in the body, the patients were further treated with systemic chemotherapy with or without biological therapy administered by an oncologist.

\section{Results}

The PVE and subsequent HSC were without any complications. Out of five administrations of autologous HSC performed so far in combination with the PVE, a sufficient hypertrophy of FLRV occurred in all five patients in the interval between 2-4 weeks following the PVE with administration of HSC (Fig. 2). Nevertheless, a significant increase of the tumour volume occurred in one female patient with hepatocellular carcinoma together with increase of FLRV, which hampered the liver resection (Figs 3, 4). Then, the patient was treated only symptomatically. Severe intraabdominal adhesions of a linitis plastica type in another patient

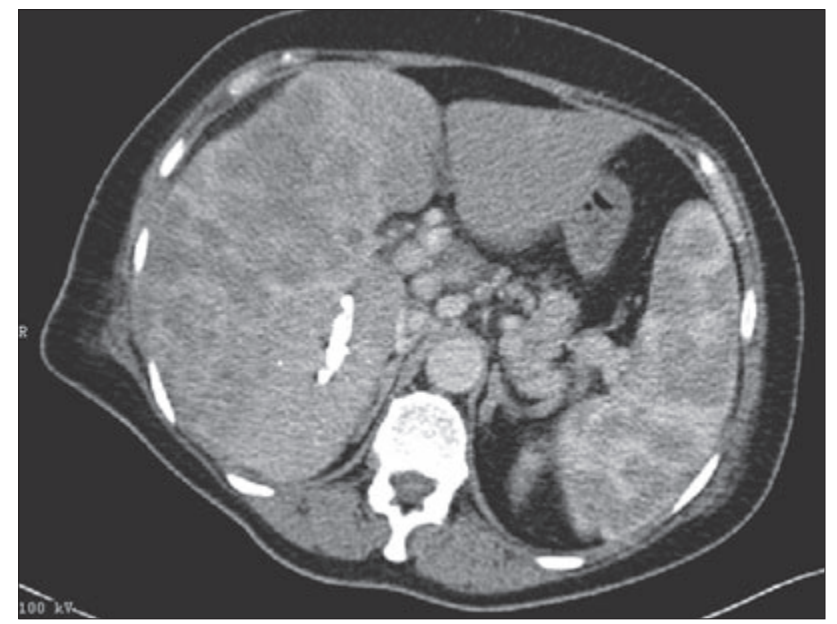

Fig. 4. Progression of HCC in the right liver lobe three weeks following the PVE and application of HSC.

meant that liver surgery was impossible and the patient continued to be treated by an oncologist. A radical liver resection (R0) was performed in three patients with colorectal cancer metastases (right-sided hepatectomy twice, extended right-sided hepatectomy once). Then, the patients were treated with a combination of adjutant chemotherapy in the FOLFOX 4 regimen and biological therapy - bevacizumab. Two patients were, 3 to 12 months after the surgery, in generally a very good condition without any signs of recurrence, and one of them experienced a solitary pulmonary metastasis 9 months following the liver resection that was treated by laser metastasectomy.

\section{Discussion}

Our previous experimental study (3) showed that a direct administration of stem cells into the contralateral liver lobe following ligation of a branch of the portal vein had a significant impact on regeneration and hypertrophy of liver parenchyma. Based on this experience and long-term clinical experience with staged procedures in the liver with the PVE, as well as due to an extensive experience with administration of HSC in haematooncological patients, we have until now used the administration of autologous HSC together with the PVE in 4 patients with secondary liver tumours and in one patient with hepatocellular carcinoma to achieve an acceleration of growth of a healthy liver lobe. The reason for support of the liver tissue growth following the PVE with HSC was based on our and literary experience that, for unclear reasons, some patients (approximately 20 to $40 \%$ ) do not experience a sufficient growth of the liver tissue following the PVE (4).

The liver is one of the few organs with its own regeneration ability. Following the liver resection, cytokines produced by both parenchymal and non-parenchymal liver cells play a key role in initiation of liver regeneration - tumour necrosis factor $\alpha$ (TNF- $\alpha$ ) and interleukin 6(IL-6), which stimulate transition of a hepatocyte from the resting $\mathrm{G} 0$ phase of cell division to the G1 phase, so called primic (5). Then, a hepatocyte is, based on other stimuli, main- 
tained in the proliferation phase by other cytokines - hepatocyte growth factor (HGF), transforming growth factor $\alpha$ (TGF- $\alpha$ ), epidermal growth factor (EGF) and insulin-like growth factor (IGF). Termination of proliferation and differentiation of the dividing hepatocytes to functionally mature hepatocytes is stimulated by transforming growth factor $\beta$ (TGF- $\beta)(3,4)$. Proliferation of hepatocytes is associated with a slight delay of non-parenchymal liver cells (cholangiocytes) proliferation and with colonisation of the Disse's spaces by new Kupffer cells. This process is finished in 3 to 4 months by a complete regeneration of the liver parenchyma (6).

In the liver tissue, there is a compartment of progenitor immature stem cells, co called "oval cells", which is probably stimulated to be differentiated in hepatocytes and cholangiocytes under physiological conditions. Their role begins most probably during the process of impairment of the common regeneration processes in the liver parenchyma when their proliferation, expansion and differentiation in the liver parenchyma cells is initiated mostly due to the effects of growth factors (HGF, EGF, TGF- $\beta$ ) $(7,8)$.

Another, very substantial source of regeneration of liver parenchyma following the liver resections during hepatic failure due to various causes are the stem cells, which are localized in other compartments of the body. The stem cells are represented in all tissues in the adult population, and blood, fat and bone tissue are their main sources for clinical needs due to their easy availability. The circulating HSCs from bone marrow are characterized by the superficial markers CD 34+ and CD 133+, glycoproteins important for mutual cell adhesion (cell-cell adhesion interaction). It is known, that these cells are largely washed out into the peripheral blood following major liver resections (9). Based on the current knowledge from regeneration of liver parenchyma we know that autologous HSC CD 34+ and CD133+ are able to differentiate into hepatocytes, Kupffer cells, cholangiocytes and endothelial cells $(10,11,12)$. So far, they have been mainly used in patients with haematological malignancies that required autologous bone marrow transplantation following the ablative chemotherapy. Recently, the focus on the use of HSC has been put on the area of therapy of liver conditions (13), cardiovascular (14) and nervous (15) diseases and diabetes (16). The HSCs are mobilized from the bone marrow using the granulocyte colony-stimulating factor (G-CSF), which involves antagonistic effect of chemokine receptor type 4 (CXCR4) which keeps HSC in the bone marrow. The second substance that enables the mobilization of HSC from bone marrow is plerixafor, a CXCR4 antagonist. Plerixafor was primarily studied as an anti-HIV agent. Nevertheless, it has been found that CXCR4 binds the stroma-derived factor (SDF-1) and hence it makes washing out of HSC from bone marrow possible and therefore it has a synergic effects with G-CSF $(17,18,19)$.

The role of HSC in regeneration of liver parenchyma was found in the experimental study performed by Mark et al (20) in the animal model of acute liver failure caused by administration of carbon tetrachloride. Following mobilization of HSC from bone marrow using Neupogen (G-CSF, Amgen Europe B.V., Breda, The Netherlands) and Mozobil (plerixafor, Genzyme, Cambridge, USA), it was possible to find a significant periportal infiltration by HSC in the impaired liver parenchyma within 24 hours using immunostaining, which used specific CD34 antibodies compared to the group of animals without such stimulation. Nevertheless, there is lack of clinical studies focused on administration of stem cells in combination with PVE in association with liver regeneration and rather case report studies are concerned $(21,22)$. Our study represents another one in this very interesting area of clinical research, which is to find a quality stimulation mechanism for growth of a contralateral liver lobe following the PVE in primary inoperable liver tumours aimed at the early radical liver resection.

Stimulation of tumour foci not only in the liver but also possible micrometastases in the body may be a serious problem of administration of HSC. Tissue of the tumour contains a high amount of stimulating substances (growth factors, matrix-metalloproteinases, cytokines), which may cause migration of HSC into the tumour tissue where they can further stimulate tumour growth and spread. The mechanism of interaction between HSC and tumour cells has not been exactly understood so far (23). A stimulation of neoangiogenesis with immunosupression (24) and inhibition of apoptosis (25) is a possible causative mechanism. A possible mechanism that facilitates growth of liver metastasis is a differentiation of mesenchymal cells in the tumour in so called carcinoma associated fibroblast (CAF)-like cells and these cells then support growth of the tumour (26). In primary liver tumours, the association is between the stem/progenitor stem cells, which are peculiar to the liver tissue and localized in the area of terminal parts of the canals of Hering and hepatocarcinogenesis $(27,28)$. Tumour growth may be facilitated also by regenerative mechanisms of the liver tissue following the PVE when a number of growth factors, matrixmetalloproteinases, cytokines are formed in the liver, which may also stimulate the liver tissue and cause its proliferation (29, $30,31,32)$. One of our patients experienced tumour progression in the contralateral, non-embolized liver lobe despite a preservation of the standard indication and therapeutic procedure. The mechanism of tumour progression is unclear and more experience is needed regarding the type of primary or secondary tumour (33, 34), tumour size in the liver parenchyma, interval between the PVE and administration of HSC and subsequent liver resection and other factors, mostly laboratory parameters to determine the risk of tumour progression following the PVE with administration of HSC. A compensatory increased arterial blood flow to the liver following the PVE, known as "hepatic arterial buffer response", which may paradoxically cause a higher blood supply of the tumour foci in the liver, will probably play a role $(35,36,37,38)$.

Our group of patients who were administered HSC following the PVE is small, but we may conclude that the selected method of acquiring and subsequent administration of HSC is suitable for clinical practice. Compared to the PVE in patients with primarily inoperable liver tumour alone, the combination of the PVE with HSC will probably be beneficial for the patient regarding faster stimulation of the growth of FRLV and an earlier radical liver resection, which is important for oncosurgery therapy. However, the danger of acceleration of tumour growth and spread in the liver and body in these patients remains an unsolved question. Currently, there is a prospective randomized study in progression at our site, which is to answer these questions. 


\section{References}

1. Ishak K, Baptista A, Bianchi L et al. Histological grading and staging of chronic hepatitis. J Hepatol 1995; 22: 696-699.

2. Brunt EM, Janney CG, Di Bisceglie AM, Neuschwander-Tetri BA, Bacon BR. Nonalcoholic steatohepatitis: a proposal for grading and staging the histological lesions. Am J Gastrenterol 1999; 94: 2467-2474.

3. Liska V, Slowik P, Eggenhofer $\mathbf{E}$ et al. Intraportal injection of porcine multipotent mesenchymal stromal cells augments liver regeneration after portal vein embolization. In vivo 2009; 23: 229-236.

4. Sánchez A, Fabregat I. Growth factor-and cytokine-driven pathways governing liver stemness and differentiation. World J Gastroenterol 2010; 16: $5148-5161$.

5. Clavien PA. Malignant liver tumors. Third Edition, Chichester: Wiley-Blackwell Publ., 2010.

6. Michalopoulos GK. Liver regeneration. J Cell Physiol 2007; 213: 286-300.

7. Duncan AW, Dorell C, Grompe M. Stem cells and liver regeneration. Gastroenterology 2009; 137: 466-481.

8. Ribeiro MAF. Liver regeneration, stem cells and beyond. World J Gastrointest Surg 2009; 1: 6-7.

9. De Silvestro G, Vicarioto M, Donadel C. Mobilization of peripheral blood hematopoietic stem cells following liver resection surgery. Hepatogastroenterology 2004; 51: 805-810.

10. Bryder D, Rossi DJ, Weissman IL. Hematopoietic stem cells: the paradigmatic tissue-specific stem cell. Am J Pathol 2006; 169: 338-346.

11. Kung JWC, Forbes SJ. Stem cells and liver repair. COBIOT 2009; 20: $1-7$.

12. Cantz T, Manns MP, Ott M. Stem cells in liver regeneration and therapy. Cell Tissue

13. Res 2008; 331: 271-282.

14. Russo FP, Parola M. Stem and progenitor cells in liver regeneration and repair. Cytotherapy 2011; 13: 135-144.

15. Wang JS, Shum-Tim D, Chedrawy E. The coronary delivery of marrow stromal cells for myocardial infarction: Pathophysiologic and therapeutic implications. J Thorac Cardiovasc Surg 2001; 122: 699-705.

16. Ben-Hur T, Idelson M, Khaner H. Transplantation of human embryonic stem cell- derived neural progenitors improves behavioral deficit in parkinsonian rats. Stem Cells 2004; 22: 1246-1255.

17. Burke ZD, Thowfeequ S, Peran M, Tosh D. Stem cells in the adult pancreas and liver. Biochem J 2007; 404: 169-178.

18. Uy GL, Retting MP, Cashen AF. Plerixafor, a CXCR4 antagonist for the mobilization of hematopoietic stem cells. Expert Opin Biol Ther 2008; 8: 1797-1804.

19. Fausto N, Campbell JS, Riehle KJ. Liver regeneration and repair: hepatocytes, progenitor cells, and stem cells. Hepatology 2004; 39: 14771487.

20. Gordon MY, Levicar N, Pai M et al. Characterization and clinical application of human CD 34+ stem/progenitor cell populations mobilized into blood by granulocyte colony-stimulating factor. Stem Cells 2006; 24: $1822-1830$.

21. Mark AL, Sun Z, Warren DS et al. Stem cell mobilization is life saving in an animal model of acute liver failure. Ann Surg 2010; 252: 591-596.
22. Wang X, Ge S, Mc Namara G. Albumin-expressing hepatocyte-like cells develop in the liver sofimine-deficient mice that received transplant of highly purified human hematopoietic stem cells. Blood 2003; 101: 4201-4208.

23. Kallis YN, Alison MR, Forbes SJ. Bone marrow stem cells and liver disease. Gut 2007; 56: 716-724.

24. Shinagawa K, Kitadai Y, Tanaka $M$ et al. Mesenchymal stem cells enhance growth and metastasis of colon cancer. Int J Cancer 2010; 127 : 2323-2333.

25. Zhu W, Xu W, Jiang R et al. Mesenchymal stem cells derived from bone marrow favor tumor cell growth in vivo. Exp Mol Pathol 2006; 80: 267-274.

26. Ramasamy R, Lam EW, Soeiro I et al. Mesenchymal stem cells inhibit proliferation and apoptosis of tumor cells: impact on in vivo tumor growth. Leukemia 2007; 21: 304-310.

27. Harun N, Nikfarjam M, Muralidharan V, Christophi Ch. Liver regeneration stimulates tumor metastases. J Surg Res 2007; 138: 284-290.

28. Mishra PJ, Mishra PJ, Humeniuk R et al. Carcinoma- associated fibroblast- like differentiation of human mesenchymal stem cells. Cancer Res 2008; 68: 4331-4339.

29. Peng N, Li L, Cai X, Tan S, Wu T. Liver stem/progenitor cells in the canals of Hering: Cellular origin of hepatocellular carcinoma with bile duct tumor thrombi? Stem Cell Rev Rep 2010; 6: 579-584.

30. Tanaka S, Yamamoto T, Tanaka H, Kodai S, Ogawa M, Ichikawa T. Potentiality of combined hepatocellular and intrahepatic cholangiocellular carcinoma originating from hepatic precursor cell: imunohistochemical evidence. Hepatol Res 2005; 32: 52-57.

31. Yokoyama Y, Nagino M, Nimura Y. Mechanisms of hepatic regeneration following portal vein embolization and partial hepatectomy: A review. World J Surg 2007; 31: 367-374.

32. Kisseleva T, Gigante E, Brenner D. Recent advances in liver stem cell therapy. Curr Opin Gastroenterol 2010; 26: 395-402.

33. Ma S, Chan KW, Hu L, Lee TK, Wo JY, Ng IO. Identification and characterization of tumorigenic liver cancer stem/progenitor cells. Gastroenterol 2007; 132: 2542-2556.

34. Peng N, Li L, Qin X et al. Evaluation of risk factors and Clinicopathologic features for intrahepatic cholangiocarcinoma in Southern China: A possible role of hepatitis B virus. Ann Surg Oncol 2011; 18: 2210-2217.

35. Lu XY, Xi T, Lau WY et al. Hepatocellular carcinoma expressing cholangiocyte phenotype is a novel subtype with highly aggressive behavior. Ann Surg Oncol 2011; 18: 2210-2217.

36. Esch JS II, Knoefel WT, Klein M et al. Portal application of autologous CD 133+ bone marrow cells to the liver: A novel concept to support hepatic regeneration. Stem Cells 2005; 23: 463-470.

37. Hayashi S, Baba Y, Ueno K. Acceleration of primary liver tumor growth rate in embolised hepatic lobe after portal vein embolisation. Acta Radiol 2007; 48: 721-727.

38. Krizkova S, Zitka O, Masarik M, Adam V, Stiborova M, Eckschlager T, Hubalek J, Krizek R. Clinical importance of matrix metalloproteinases. Bratisl Lek Listy 2011; 112: 435-440.

39. Pachos KA, Bird NC. Liver regeneration and its impact on post-hepatectomy metastatic tumor recurrence. Anticancer Res 2010; 30: 2161-2170.

Received October 11, 2011. Accepted November 30, 2011. 